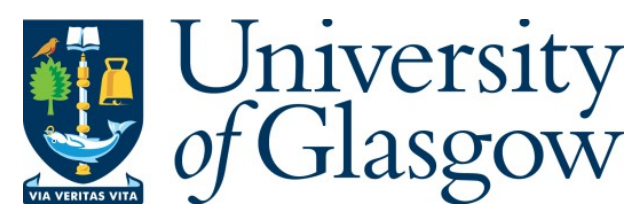

Katranaras, E., Imran, M. A., Dianati, M., and Tafazolli, R. (2014) Green inter-cluster interference management in uplink of multi-cell processing systems. IEEE Transactions on Wireless Communications, 13(12), pp. 6580-6592.

There may be differences between this version and the published version. You are advised to consult the publisher's version if you wish to cite from it.

http://eprints.gla.ac.uk/135470/

Deposited on: 26 January 2017

Enlighten - Research publications by members of the University of Glasgow http://eprints.gla.ac.uk 


\title{
Green Inter-Cluster Interference Management in Uplink of Multi-Cell Processing Systems
}

\author{
Efstathios Katranaras, Member, IEEE, Muhammad Ali Imran, Senior Member, IEEE, \\ Mehrdad Dianati, Senior Member, IEEE and Rahim Tafazolli, Senior Member, IEEE \\ Institute for Communication Systems (ICS), University of Surrey, UK \\ Email: \{e.katranaras, m.imran, m.dianati, r.tafazolli\}@surrey.ac.uk
}

\begin{abstract}
This paper examines the uplink of cellular systems employing base station cooperation for joint signal processing. We consider clustered cooperation and investigate effective techniques for managing inter-cluster interference in order to improve users' performance in terms of both spectral and energy efficiency. We use information theoretic analysis to establish general closed form expressions for the system achievable sum rate and the users' Bit-per-Joule capacity while adopting a realistic user device power consumption model. Two main intercluster interference management approaches are identified and studied, i.e. through: 1) spectrum re-use; and 2) users' power control. For the former case, we show that isolating clusters by orthogonal resource allocation is the best strategy. For the latter case, we introduce a mathematically tractable user power control scheme and observe that a green opportunistic transmission strategy can significantly reduce the adverse effects of inter-cluster interference while exploiting the benefits from cooperation. In order to compare the different approaches in the context of realworld systems and evaluate the effect of key design parameters on the users' energy-spectral efficiency relationship, we fit the analytical expressions into a practical macrocell scenario. Our results demonstrate that significant improvement in terms of both energy and spectral efficiency can be achieved by energy-aware interference management.
\end{abstract}

Index Terms-Land mobile radio cellular systems, Multi-Cell Processing, Green wireless communications, User-side energy conservation, Interference management, Information theory.

\section{INTRODUCTION}

Energy consumption of communication systems has recently become an important issue [1]. Particularly, wireless access networks have been an active subject of interest due to their current and (most importantly) forecasted significant contribution to the overall energy consumption of communication networks [2]. This has led to extensive research on "green" techniques for wireless communications to minimise their carbon footprint as well as the energy conservation for economical (lower energy costs) and practical (increased battery life in mobile devices) benefits [3], [4], [5]. While most recent studies focus on the operational energy conservation of radio Base Stations (BSs), the significance of total energy consumption or Energy Efficiency (EE) at terminal ends, due to network activity at both uplink and downlink, shall not be belittled [6]. Especially in uplink, EE at terminal ends can be straightforwardly improved by managing their transmit power. The explosive growth of user terminals (currently in the order of billions [7]) and especially of battery draining smart phones downloading content from the Internet, renders the augmentation of the overall end user devices' EE a very important factor for achieving "holistic green" communication systems, i.e. from both network and user devices perspective.

On the other hand, the need of new services, emerging from the wide-spreading use of mobile internet, retains the trend of exploding data volume and throughput demand in cellular industry. Essentially, this demand translates into high Spectral Efficiency (SE) needs requiring aggressive radio spectrum usage [8]. Unavoidably, future deployments will face critically increased levels of Inter-Cell Interference (ICI) which, if left uncontrolled, leads into significant losses in throughput and fairness degradation. To this end, multi-cell cooperation is regarded as a key technique for ICI management in cellular systems [9]. In particular, cooperation through multi-cell joint signal processing, where transmit (in downlink) or receive (in uplink) information is exchanged among cells, provides the potential of even exploiting ICI as a diversity signal [10], [11]. The resulting Distributed Multiple-Input-Multiple-Output (DMIMO) system provides macro-diversity gains [12] and has the potential to boost overall $\mathrm{SE}$ as well as to provide more homogeneous distribution of user data rates [13].

Multi-cell cooperation, although not a newly introduced concept, has become only currently a very active field of research for practical implementation under the label of "Coordinated Multi-Point" in LTE-Advanced standards [14]. Thus, while earlier studies focused on the theoretical SE gains in global cooperation systems, where cooperation among all cells in a system is assumed possible, more recently, implementation related issues have been examined (e.g. see [15], [16], [17], [18], [19], [9]). These practical issues mainly regard the extra effort needed for signal processing to enable BS cooperation as well as the limitations of the backhaul links connecting cooperating BSs. It has become evident that a limited number of BSs can cooperate in practical systems for affordable overhead, complexity and energy consumption burden. In that regard, this paper's focus is on clustered cooperation, where multiple sets of cells in a system form independent cooperation clusters, which is essentially a more practically realisable scenario.

However, clustered cooperation introduces undesired InterCluster Interference (ICLI) into the system. Since there is no coordination available between the clusters, ICLI can in principle be managed in a similar way as ICI is mitigated in conventional uncoordinated networks. Focusing in the uplink, the various ICI mitigation techniques are generally classified 
into [20]: 1) avoidance, i.e. by re-use of time/frequency resources; 2) randomisation i.e. by averaging the interference at BSs and making ICI appear as additional background Additive White Gaussian Noise (AWGN); 3) cancellation, i.e. by regenerating the interfering signals at BSs and subsequently subtract them from the desired signal; and 4) power control at User Terminals (UTs). Regarding ICLI management, all aforementioned approaches can be essentially applied; however, cancellation techniques are quite impractical since the generally large amount of out-of-cluster interfering UTs will add extreme processing complexity to BSs. On the other hand, randomization techniques can be combined with either avoidance or power control techniques to mitigate any residual ICLI. Therefore, there exist two main practical ways to reduce ICLI in uplink (described in more detail within Section IV): a) through effective spectrum re-use among clusters and; b) through effective power control of user devices. In this work, both approaches are analysed and compared with the benchmark scenario of single-cell processing and the worstcase scenario of total ICLI allowance where all inter-cluster signals are simply tolerated without any ICLI management technique taking place.

The main objective of this paper is to identify if and under which circumstances ICLI management techniques can improve the EE-SE relationship of all active UTs during uplink in multi-cell processing systems by providing a good balance between the harmful effect due to ICLI and the benefits originating from BSs cooperation. In general, EESE relationships are considered as important performance measures for deployment and operation of future wireless access systems and it has in fact been shown that in practice they follow a non-monotonic trend [21]. In this work, we are interested in energy-aware solutions when the throughput performance of the system is still of high priority, i.e. we want to optimize system SE while harvesting potential EE gains for UTs. Uplink activities are proved to be of equal importance to downlink activities in terms of energy consumption at user radio modules for services or applications where either more (e.g. cellular phone calls, text messaging, emailing, internetbased audio/video calls) or less (e.g. web browsing, file download, gaming) data have to be transferred in the uplink [6]. Thus, the investigation of techniques to improve SE and $\mathrm{EE}$ in uplink is also imperative. To obtain useful insights on the potential performance gains of the different ICLI management techniques we characterise the system SE and overall UTs' EE, realised in uplink, considering the system achievable sum rate and the overall users' Bit-per-Joule capacity, respectively. Thus, we formulate general closed form SE and EE expressions based on an information theoretic analysis. To obtain a realistic view on the users EE-SE relationship we need to get a full picture of the total consumed power at the UTs. To this end, we consider a general user device consumption model including circuit power dissipation as proposed in [22].

The main contribution of this work lies on the detailed analysis of the different ICLI management approaches, in terms of both system SE and overall UTs' EE in uplink. We first show that the best spectrum re-use strategy is to completely isolate clusters, i.e. fully eliminate ICLI. On the other hand, for UT power control approaches, we introduce a mathematically tractable power control scheme and we observe that by employing an opportunistic transmission strategy, both the adverse effects of inter-cluster interference and high energy consumption at user devices can be significantly reduced while the positive impacts of intra-cluster joint processing can still be exploited. Furthermore, we evaluate the analytical expressions in the context of a real-world network by interpreting them into a practical macrocell deployment scenario. Our results reveal that significant gains in both EE and SE can be yielded through appropriate energy-aware ICLI management. In addition, we identify and analyse the effect of various key design parameters of the practical multi-cell system, such as inter-site distance, number of served UTs per cell and cooperation cluster size among others, that affect the overall UTs' EE-SE relationship.

The rest of the paper is organised as follows. First, Section II introduces the system model. In section III we derive the general analytical expressions characterising the SE and EE of the clustered cooperative network in uplink and we formulate the general optimization problem for UTs' performance. Section IV presents the main approaches for ICLI management and provides performance formulations for each case. Particularly, subsection IV-A deals with ICLI management through spectrum re-use while subsection IV-B introduces a tractable model to mitigate ICLI through UT power control. Section V provides numerical results interpreting the theoretical analysis into the context of a real-world network along with insightful observations and evaluation on overall UTs' EE-SE relationship. Finally, concluding remarks are given in section VI.

For the mathematical formulations in the paper, the following hold. Scalars, variables, column vectors, matrices and sets are denoted by capital italic, lowercase italic, bold lowercase, bold capital and calligraphic letters respectively. $\mathbf{1}_{n}$ denotes the all ones column vector of size $n$ while $\mathbf{I}_{n}$ denotes an $n \times n$ identity matrix. Moreover, $(\cdot)^{*}$ denotes the complex conjugate, $(\cdot)^{\dagger}$ denotes the conjugate transpose matrix, $|\cdot|$ denotes the norm of a complex scalar, $\mathbb{E}[\cdot]$ stands for the expectation of the respective scalar or matrix and $\boldsymbol{\Lambda}_{(\cdot)}$ stands for the covariance matrix of the respective vector. $\mathcal{C N}$ represents a complex Gaussian distribution. $\mathcal{Z}_{n}$ is the finite set of integers modulo $n$. Symbols $\triangleq$ and $\underset{n \rightarrow \infty}{\stackrel{a . s .}{\longrightarrow}}$ stand for "is defined as" and "converges almost surely when $n$ tends to infinity", respectively.

\section{SySTEM MODEL}

This section introduces the system model considered in this paper including the main assumptions on the network setup, the propagation and the channel model.

\section{A. System Deployment}

The uplink of a linear cellular grid, as shown in Fig. 1, is considered in this paper, similar to the one presented in [23]. The linear model has been extensively used in theoretical works as it provides very accurate insights on the performance of most practical cellular networks (e.g. see [11], [24], [25]) while keeping the mathematical analysis tractable. It is also rather suitable for describing several real-world scenarios, e.g. 


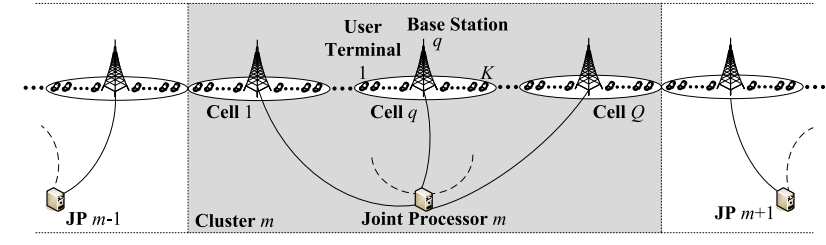

Fig. 1. Multi-cell system with clustered cooperation.

highways, train lines or railway networks, satellite cellular networks. Thus, we consider a system comprising $N$ cells, each served by a single antenna BS positioned at its centre. Note that since each BS forms a single cell, both terms will be used interchangeably hereafter. Thus, when for example it is mentioned that a UT is in cell $q$, this means that this UT is associated with the $q^{\text {th }}$ BS forming this specific cell. The InterSite Distance (ISD) between consequent BSs is assumed same for any two BSs. Moreover, $K$ single antenna UTs are at any time associated with each BS. We consider the UTs uniformly positioned across the grid of their associated cell; the uniform positioning renders the mathematical analysis of the system more tractable while providing a precise approximation of the average effect from randomly positioned UTs over a reasonable amount of system instances [26]. It is noted that both system geometry and UT spatial distribution can be adjusted to any other higher complexity topology scenario.

Furthermore, we consider multiple subsequent BSs forming cooperation clusters. Cooperation among BSs is limited only to those belonging to the same cluster; hence, a Joint Processor (JP) in each cluster of cells can be considered to jointly decode all received signals from UTs in that cluster. We consider that each of the $M$ total formed clusters comprises $Q$ BSs with $Q \ll N$. It is further assumed that cooperating BSs are perfectly synchronised in frequency and time (e.g. by using a global positioning system), JPs have perfect knowledge of the channels between UTs and BSs in their assigned cluster (e.g. through an efficient estimation process achieving accurate channel state information at BSs, extracted through UT transmissions), while unlimited, delayless and error-free information exchange is taking place between JPs and their assigned cluster BSs (e.g. through a high-speed fiber backhaul network) [27]. Thus, each cluster can be essentially viewed as a DMIMO system. Moreover, a wideband medium access scheme is considered, as defined in [11], where at any time all active UTs in the cluster (assumed as many as the BSs in the cluster) share non-orthogonally the whole system bandwidth and their signals get perfectly decoded at the cooperating BSs, i.e. no intra-cluster interference is considered.

\section{B. Channel Model}

For the channel model we consider the complex baseband of linear and memoryless flat fading channels. Hence, the received symbol $y^{m, q}$ at the $\mathrm{BS}$ in any cell $q \in \mathcal{Z}_{Q}$ of any cluster $m \in \mathcal{Z}_{M}$, at any time, can be viewed as the sum of the simultaneously transmitted symbols $x_{m, \dot{q}, k}$ from all the $Q K$ UTs in the same cluster of cells plus the interfering symbols $x_{\dot{m}, \dot{q}, k}$ from UTs in cells outside the cluster of interest, $\forall \dot{q}, \dot{m} \in \mathcal{Z}_{Q}, \mathcal{Z}_{M}$ respectively, all appropriately scaled by channel attenuation coefficients, plus the noise $n^{m, q}$ realised at the reference $\mathrm{BS}$ :

$$
\begin{aligned}
y^{m, q}=\sum_{\dot{q}=1}^{Q} & \sum_{k=1}^{K} \varsigma_{m, \dot{q}, k}^{m, q} g_{m, \dot{q}, k}^{m, q} x_{m, \dot{q}, k} \\
& +\sum_{\dot{m} \neq m} \sum_{\dot{q}=1}^{Q} \sum_{k=1}^{K} \varsigma_{\dot{m}, \dot{q}, k}^{m, q} g_{\dot{m}, \dot{q}, k}^{m, q} x_{\dot{m}, \dot{q}, k}+n^{m, q}
\end{aligned}
$$

where $\varsigma_{\dot{m}, \dot{q}, k}^{m, q}$ and $g_{\dot{m}, \dot{q}, k}^{m, q}$ denote the path loss and flat fading coefficient, respectively, experienced in the transmission path between BS $q$ in cluster $m$ and UT $k \in \mathcal{Z}_{K}$ in cell $\dot{q}$ of cluster $\dot{m}$. Path loss coefficients are determined based on a powerlaw path loss model [28]: $\varsigma_{\dot{m}, \dot{q}, k}^{m, q}=\left(1+d_{\dot{m}, \dot{q}, k}^{m, q}\right)^{-\eta / 2}$ with $\eta$ denoting the path loss exponent and $d_{\dot{m}, \dot{q}, k}^{m, q}$ defined as the distance along the direct path. Each UT is assumed to have an average transmit power constraint, i.e. $\mathbb{E}\left[x x^{\star}\right] \leq P_{\max }$. Moreover, flat fading coefficients are independent identically distributed (i.i.d.), complex circularly symmetric (c.c.s.) Gaussian random variables, i.e. $g \sim \mathcal{C N}(0,1)$. Furthermore, $n^{m, q}$ denotes the AWGN realised at any BS $m$ in cluster $q$ with power $\sigma^{2}$, i.e. $n \sim \mathcal{C N}\left(0, \sigma^{2}\right)$.

ICLI can be assumed a sum of numerous complex Gaussian inputs and thus, can be viewed as an, independent from the AWGN, additional noise component at the BSs. Hence, the power of the undesired symbol $z^{m, q}$, i.e. the sum of ICLI and AWGN, can be given by:

$$
\begin{aligned}
\mathbb{E}\left[z^{m, q}\left(z^{m, q}\right)^{*}\right]=\sigma^{2}+\sum_{\dot{m}} \sum_{\dot{q}=1}^{Q} \sum_{k=1}^{K} \\
\mathbb{E}\left[\left(\varsigma_{\dot{m}, \dot{q}, k}^{m, q} g_{\dot{m}, \dot{q}, k}^{m, q} x_{\dot{m}, \dot{q}, k}\right)\left(\varsigma_{\dot{m}, \dot{q}, k}^{m, q} g_{\dot{m}, \dot{q}, k}^{m, q} x_{\dot{m}, \dot{q}, k}\right)^{*}\right]
\end{aligned}
$$

We note that the treatment of ICLI as an additional noise component is not the optimal approach for achieving capacity in any system; for example, in the simple interference channel case, the optimal approach depends on the strength of the interference links and considering interference as noise is proved to be optimal only for very weak interference [29]. However, this is a fairly valid assumption for practical macrocell systems which will constitute the focus of this work; in such systems the average interference originating from UTs in adjacent clusters, when compared to the average desired received power in the cluster of interest, can be considered weak enough.

\section{Spectral AND ENERGy EFFICIENCY}

In this section we characterise the system SE and the overall UTs' EE, realised in uplink, to establish general closed form solutions for each individual metric as well as for their relationship. To this end, we first regard the per-cluster ergodic achievable sum rate (sum of all UT rates in the cluster) to evaluate the SE of the system. We use an information theoretic analysis for its derivation and provide a closed form approximation formula. We then define the BS contributing rate metric, which can stand as a useful measure for clustered systems. On the other hand, user-side EE is characterized 
by the Bit-per-Joule Capacity, focusing on the efficient use of UTs' energy to transmit their data. We adopt a realistic UT power consumption model and discuss how overall UTs' EE gains can be realised in the uplink of any cooperative system where multiple cells exchange receive information to manage ICI. Finally, we formulate the general analysis for the performance optimization of the system.

\section{A. System Spectral Efficiency: The Ergodic Sum Rate}

For the performance analysis we assume that all clusters are identical, e.g. a circular model can be considered to avoid system edge effects [30]. In that case, results from a single cluster analysis become valid for the whole system. Thus, the baseband cluster channel model, for any cluster $m$, can be expressed in matrix form as:

$$
\mathbf{y}^{m}=\mathbf{H}^{m} \mathbf{x}^{m}+\mathbf{H}_{\mathbf{I}}^{m} \mathbf{x}_{\mathbf{I}}^{m}+\mathbf{n}^{m}=\mathbf{H}^{m} \mathbf{x}^{m}+\mathbf{z}^{m},
$$

where $\mathbf{y}^{m} \triangleq\left[y^{m, 1} \ldots y^{m, Q}\right]^{T}$ stands for the $Q \times 1$ received symbol vector by all BSs in the cluster, jointly processed at the corresponding JP; $\mathbf{x}^{m} \triangleq\left[\mathbf{x}_{m, 1}{ }^{T} \ldots \mathbf{x}_{m, Q}\right]^{T}$ is the $Q K \times 1$ transmitted symbol vector from all UTs in the cluster, with $\mathbf{x}_{m, q} \triangleq\left[x_{m, q, 1} \ldots x_{m, q, K}\right]^{T}$ denoting the concatenation of the transmitted symbols from the $K$ UTs in cell $q ; \mathbf{H}$ is the overall $Q \times Q K$ cluster channel gain matrix comprising the respective channels' path loss and flat fading coefficients; finally, $\mathbf{z}^{m}=\mathbf{H}_{\mathbf{I}}^{m} \mathbf{x}_{\mathbf{I}}^{m}+\mathbf{n}^{m} \triangleq\left[z^{m, 1} \ldots z^{m, Q}\right]^{T}$ denotes the $Q \times 1$ noise plus interference vector, with $\mathbf{x}_{\mathbf{I}}^{m}$ representing the $(M-1) Q K \times 1$ transmitted symbol vector from all UTs outside the cluster, $\mathbf{H}_{\mathbf{I}}^{m}$ standing for the $Q \times(M-1) Q K$ respective channel gain matrix and $\mathbf{n}^{m}$ being the the $Q \times 1$ AWGN vector. Since all clusters are considered identical we omit the cluster superscript index in system matrices hereafter. Cluster sum rate is bounded by the joint mutual information between the UTs and BSs in cluster $m$ and thus, assuming that the UTs apply independent, complex Gaussian codebooks and that channel $\mathbf{H}$ is fully known at the cluster BSs, the ergodic achievable cluster sum rate (or capacity) is given by:

$$
C \triangleq W \mathbb{E}_{g}\left[\log _{2}\left(\frac{\operatorname{det}\left(\mathbf{H} \boldsymbol{\Lambda}_{\mathbf{x}} \mathbf{H}^{\dagger}+\boldsymbol{\Lambda}_{\mathbf{z}}\right)}{\operatorname{det}\left(\boldsymbol{\Lambda}_{\mathbf{z}}\right)}\right)\right] \text { bits/sec, }
$$

where $\boldsymbol{\Lambda}_{\mathbf{z}}$ is the noise plus interference covariance matrix, $W$ is the system bandwidth and the expectation is taken over all the system fading realizations. Considering separately the numerator and denominator inside the logarithmic function in (4), Jensen's inequality (i.e. $\mathbb{E}[\phi(\mathbf{A})] \leq \phi(\mathbb{E}[\mathbf{A}])$, for any convex function $\phi$ and matrix $\mathbf{A}$ ) [31], can be applied (since - log det is a convex function [32]), and in fact reduce to equality; for large enough number of users per cell, the dimensionality of matrices in (4) becomes large, with improved eigenvalue distribution, rendering safe to assume them converging to deterministic matrices according to the law of large numbers [11]. In that case:

$$
\begin{aligned}
\mathbb{E}_{g}\left[\log _{2} \operatorname{det}\left(\mathbf{H} \boldsymbol{\Lambda}_{\mathbf{x}} \mathbf{H}^{\dagger}+\boldsymbol{\Lambda}_{\mathbf{z}}\right)\right] \\
\stackrel{K \rightarrow \infty}{\longrightarrow} \log _{2} \operatorname{det} \mathbb{E}_{g}\left[\mathbf{H} \boldsymbol{\Lambda}_{\mathbf{x}} \mathbf{H}^{\dagger}+\boldsymbol{\Lambda}_{\mathbf{z}}\right]
\end{aligned}
$$

and

$$
\mathbb{E}_{g}\left[\log _{2} \operatorname{det}\left(\boldsymbol{\Lambda}_{\mathbf{z}}\right)\right] \stackrel{K \rightarrow \infty}{\longrightarrow} \log _{2} \operatorname{det} \mathbb{E}_{g}\left[\boldsymbol{\Lambda}_{\mathbf{z}}\right]
$$

Thus, cluster capacity converges as:

$$
\begin{aligned}
C=W \mathbb{E}_{g}\left[\log _{2}\left(\frac{\operatorname{det}\left(\mathbf{H} \boldsymbol{\Lambda}_{\mathbf{x}} \mathbf{H}^{\dagger}+\boldsymbol{\Lambda}_{\mathbf{z}}\right)}{\operatorname{det}\left(\boldsymbol{\Lambda}_{\mathbf{z}}\right)}\right)\right] \\
\stackrel{K \rightarrow \infty}{\longrightarrow} W \log _{2}\left(\frac{\operatorname{det} \mathbb{E}_{g}\left[\mathbf{H} \boldsymbol{\Lambda}_{\mathbf{x}} \mathbf{H}^{\dagger}+\boldsymbol{\Lambda}_{\mathbf{z}}\right]}{\operatorname{det} \mathbb{E}_{g}\left[\boldsymbol{\Lambda}_{\mathbf{z}}\right]}\right) .
\end{aligned}
$$

Note that in Section V, we verify through simulations that the above convergence stands for a tight approximation in practical systems with reasonable number of UTs per cell.

We further approximate cluster capacity while considering different transmit power at each UT. We introduce the overall UTs' transmit power profile $\mathbf{p} \triangleq\left[P_{1,1} \ldots P_{q, k} \ldots P_{Q, K}\right]^{T}$ as the vector defining the set of the transmit powers from all UTs in the cluster. Thus, by assuming independent inputs, the $Q K \times Q K$ input covariance matrix $\boldsymbol{\Lambda}_{\mathbf{x}}$ will be diagonal, constrained by the maximum UT transmit power:

$$
\mathbf{\Lambda}_{\mathbf{x}} \triangleq \mathbb{E}\left[\mathbf{x}^{m}\left(\mathbf{x}^{m}\right)^{\dagger}\right]=\mathbf{p}\left(\mathbf{1}_{Q K}\right)^{T} \leq P_{\max } \cdot \mathbf{I}_{Q K},
$$

where $P_{q, k}$ denotes the transmit power of UT $k$ in cell $q$ of any cluster. Furthermore, considering (2) and the Rayleigh nature of the flat fading, the expected noise plus interference covariance matrix will be of the form:

$$
\begin{aligned}
\mathbb{E}_{g}\left[\boldsymbol{\Lambda}_{\mathbf{z}}\right] & =\mathbb{E}_{g}\left[\mathbf{n} \mathbf{n}^{\dagger}+\mathbf{H}_{\mathbf{I}} \boldsymbol{\Lambda}_{\left.\mathbf{x}_{\mathbf{I}} \mathbf{H}_{\mathbf{I}}^{\dagger}\right]} \underset{\underset{K \rightarrow \infty}{a . s .} \operatorname{diag}}{Q \text { elements }}(\overbrace{\ldots \sigma^{2}+\sum_{\dot{m}} \sum_{\dot{q}=1}^{Q} \sum_{k=1}^{K} P_{\dot{q}, k}\left(\varsigma_{\dot{m}, \dot{q}, k}^{m, q}\right)^{2} \ldots}) .\right.
\end{aligned}
$$

By substituting (7), (8) and (9) in (4) and recalling that the determinant of a diagonal matrix is the product of its diagonal elements, we get:

$$
\begin{gathered}
C \underset{K \rightarrow \infty}{\stackrel{a . s .}{\longrightarrow}} W \log _{2} \prod_{q=1}^{Q} \\
{\left[1+\frac{\sum_{\dot{q}=1}^{Q} \sum_{k=1}^{K} P_{\dot{q}, k}\left(\varsigma_{m, \dot{q}, k}^{m, q}\right)^{2}}{\sigma^{2}+\sum_{\forall \dot{m} \neq m} \sum_{\dot{q}=1}^{Q} \sum_{k=1}^{K} P_{\dot{q}, k}\left(\varsigma_{\dot{m}, \dot{q}, k}^{m, q}\right)^{2}}\right] \triangleq R_{m} .}
\end{gathered}
$$

To comprehend the convergence in both (9) and (10) it is highlighted here that: 1) at the diagonal entries of $\mathbb{E}_{g}\left[\mathbf{H} \boldsymbol{\Lambda}_{\mathbf{x}} \mathbf{H}^{\dagger}\right]$ and $\mathbb{E}_{g}\left[\mathbf{H}_{\mathbf{I}} \boldsymbol{\Lambda}_{\mathbf{x}_{\mathbf{I}}} \mathbf{H}_{\mathbf{I}}{ }^{\dagger}\right]$, takes place the product $\mathbb{E}_{g}\left[|g|^{2}\right]=1$ and; 2) at the off-diagonal entries, we consider the fact that the expectation of the product of two different realisations of the fading coefficients $\mathbb{E}_{g}\left[g\left(g^{\prime}\right)^{*}\right]=0$, where $g \neq \dot{g}$, indicating that both $\mathbb{E}_{g}\left[\mathbf{H} \boldsymbol{\Lambda}_{\mathbf{x}} \mathbf{H}^{\dagger}\right]$ and $\mathbb{E}_{g}\left[\mathbf{H}_{\mathbf{I}} \boldsymbol{\Lambda}_{\mathbf{x}_{\mathbf{I}}} \mathbf{H}_{\mathbf{I}}^{\dagger}\right]$ converge to $Q \times Q$ diagonal matrices. In this paper, the approximated sum rate formulation in (10), i.e. $R_{m}$, is used to assess the SE of the system which stands for solidly tight approximation (as will be shown in section V). 
1) Individual BS Contribution on Sum Rate: The cluster sum rate given in (10) can be written in an equivalent condensed form:

$$
R_{m}=W \sum_{q=1}^{Q} \log _{2}\left(1+\frac{P_{\mathrm{D}}^{m, q}}{P_{\mathrm{U}}^{m, q}}\right),
$$

where $P_{\mathrm{D}}^{m, q}$ and $P_{\mathrm{U}}^{m, q}$ stand for the desired (from UTs within the region of the respective JP) and the undesired (i.e. interference plus noise as given in (2)) power received, respectively, in BS $q$ of cluster $m$. We can define the contribution of the $q^{\text {th }}$ $B S$ on cluster sum rate as:

$$
\begin{aligned}
& R_{m, q} \triangleq W \log _{2}\left(1+\operatorname{SINR}_{m, q}\right) \\
= & W \log _{2}\left(1+\frac{\sum_{\dot{q}=1}^{Q} \sum_{k=1}^{K} P_{\dot{q}, k}\left(\varsigma_{m, \dot{q}, k}^{m, q}\right)^{2}}{\sigma^{2}+\sum_{\dot{m}} \sum_{\dot{q}=1}^{Q} \sum_{k=1}^{K} P_{\dot{q}, k}\left(\varsigma_{\dot{m}, \dot{q}, k}^{m, q}\right)^{2}}\right)
\end{aligned}
$$

where $\operatorname{SINR}_{m, q} \triangleq P_{\mathrm{D}}^{m, q} / P_{\mathrm{U}}^{m, q}$ is the Signal-to-Interferenceplus-Noise-Ratio (SINR) realised at the respective BS. Note that the equality in (12) comes from the fact that $\operatorname{SINR}_{m, q}$ actually refers to the $q^{\text {th }}$ individual product term in (10). Generally, due to cluster edge effects, an uneven SINR distribution is realised among BSs in the cluster as desired (due to cooperation) and undesired (due to ICLI) average received power at each BS will highly depend on its location within the cluster. The BS contribution rate can be used as a useful figure of merit as in some way reveals the portion of the cluster sum rate that can be attributed to each individual cooperating entity; thus, it could provide important information to network operators for optimizing clustering among cells (i.e. deciding which BSs should cooperate with each other) or even for the actual deployment (e.g. installation locations) of BSs. Therefore, in Section $\mathrm{V}$, we also provide results for the individual BS contributing rates as this analysis can become a useful starting point for future research on that area.

\section{B. Overall Users' Energy Efficiency: The Bit/Joule Capacity}

To evaluate the EE of all active UTs in the network during uplink, it is important to adopt an appropriate metric that characterizes the correspondence between the consumed resources (i.e. the total energy consumed by UTs during transmission) and the attained utility (i.e. the uplink useful information exchange in Bits). Therefore, we focus on the UTs' Bit-per-Joule Capacity which is a very important metric for capacity limited future multi-media applications systems. In order to get a realistic view of the total consumed energy at UTs during transmission mode we have to consider both transmit power $\left(P_{q, k}\right)$ and circuit power $\left(P_{C, k}\right)$ at any $k^{\text {th }} \mathrm{UT}$ in any cell $q$. Circuit power represents the energy consumption of the UTs electronics and is considered independent of data rate [22]. On the other hand, transmit power of each UT will be decided by the chosen transmit power profile. In general, the power of a UT $k$ in cell $q$ can be given by:

$$
P_{q, k}^{\mathrm{TOT}}=P_{C, k}+\frac{P_{q, k}}{\epsilon_{k}}
$$

where $\epsilon_{k} \in[0,1]$ denotes the efficiency of the power amplifier at the UT. We note that in reality, the circuit powers and power amplifiers of different UTs are largely variant. However, to analytically model the overall UTs' EE, without any loss of generality, we normalise the circuit power of any UT to an average $P_{C}$ value and assume ideal power amplifiers, i.e. $\epsilon_{k}=\epsilon=1, \forall k$; the UTs' power differences, in the sense of various circuit power and amplifier efficiency values perceived at different user devices, can be simply absorbed into the channel coefficients or the noise variances in equation (10). Thus, average UT EE, $U$, can be formulated as:

$$
U \triangleq \frac{R_{m}}{\sum_{q} \sum_{k} P_{q, k}^{\mathrm{TOT}}}=\frac{R_{m}}{Q K P_{C}+\sum_{q} \sum_{k} P_{q, k}} \text { bits/Joule. }
$$

Eventually, it becomes obvious from (14) that the average UT EE realised at any cooperative system depends on two factors: 1) the sum rate achieved and; 2) the sum power used by UTs. Thus, there exist two types of overall UTs' EE gain: 1) the $S E$-related $E E$ gain, which is in fact equivalent to the $S E$ gain realised due to BSs cooperation and 2) the Power-related EE gain which arises from any power usage reductions at UTs. Note that the Power-related EE gain is more implicitly linked to UTs' green operation as it has to do with the reduction of the total energy consumed over a time period and thus, the energy cost reduction.

\section{Performance Optimisation Problem}

We formulate now the UTs' performance optimization problem. In (10) and (14), we have derived analytical formulas to calculate system SE and overall UTs' EE, respectively, as functions of: transmit power profile $\mathbf{p}$; cluster size $Q$; number of UTs per cell $K$; topology and propagation system setup (ISD, $\eta$ ); and system bandwidth $W$. Furthermore, EE is also a function of UTs' circuit power $P_{C}$. The general optimization problem can be expressed as:

$$
\begin{aligned}
& {\left[\hat{\mathbf{p}}, \hat{Q}, \hat{K}, \mathrm{ISD}, \hat{\eta}, \hat{W}, \hat{P_{C}}\right]=} \\
& \left.\arg \max _{\mathbf{p}, Q, K, \mathrm{ISD}, \eta, W, P_{C}} F\left(\mathbf{p}, Q, K, \mathrm{ISD}, \eta, W, P_{C}\right)\right|_{\mathcal{X}},
\end{aligned}
$$

where $\mathcal{X}$ represents the set of all system constraints and $F$ can be either the SE or EE performance metric. Expression (15) stands for a high-dimensional optimization problem, nonconvex due to the discreteness of most optimization parameters and thus, difficult to solve. We can, however, simplify the problem by fixing certain parameters.

As already mentioned, our interest lies on optimizing the overall UTs' EE-SE relationship by achieving the maximum system SE while at the same time identifying the possible UTs' EE gains that can be attained. Therefore, in the following, we define and investigate the optimal transmit power profile, $\mathbf{p}^{\star}$, such that the cluster sum rate $R_{m}$ is maximized under the given system set of power constraints and parameters $\left(Q, K\right.$, ISD, $\left.\eta, W, P_{C}\right)$. Along with $P_{\max }$, we consider a minimum transmit power constraint, $P_{\min }$, which represents the minimum average power needed at any UT during transmission mode to perform operations like signaling 
and/or other emergency communications. Thus, we determine:

$$
\mathbf{p}^{\star}=\arg \max _{\mathbf{p} \in\left\{\mathbf{p} \mid P_{\min } \leq P_{q, k} \leq P_{\max }, \forall(q, k)\right\}} R_{m}(\mathbf{p}),
$$

where the set $\left\{\mathbf{p} \mid P_{\min } \leq P_{q, k} \leq P_{\max }, \forall(q, k)\right\}$ stands for the feasible set of transmit power vectors under the specific power requirements posed. The corresponding EE-SE relationship will then be given as:

$$
U\left(\mathbf{p}^{\star}\right) \triangleq \frac{R_{m}\left(\mathbf{p}^{\star}\right)}{\sum_{q} \sum_{k} P_{q, k}^{\mathrm{TOT}}\left(\mathbf{p}^{\star}\right)} .
$$

Now, we can make some further observations regarding the UTs' EE-SE relationship. In the clustered cooperative system, two main "forces" decide the overall experienced SE gain; in one hand, there exists a positive effect due to ICI management through cooperation within the cluster (represented by the desired received power $P_{\mathrm{D}}^{m, q}$ in (11)); in the other hand, there is a negative effect due to ICLI (included in the undesired received power $P_{\mathrm{U}}^{m, q}$ in (11)). It becomes obvious from (10) that both $P_{\mathrm{D}}^{m, q}$ and $P_{\mathrm{U}}^{m, q}$ are functions of the UTs' transmit power profile, following the same convexity, i.e. for any two profiles $\mathbf{p}_{1} \succeq \mathbf{p}_{2}$, choosing $\mathbf{p}_{1}$ instead of $\mathbf{p}_{2}$ will increase both $P_{\mathrm{D}}^{m, q}$ and $P_{\mathrm{U}}^{\bar{m}, q}$ (and essentially, both the cooperation and ICLI effect) and vice-versa. Furthermore, the EE gain is also directly a function of the UTs' transmit power profile. Therefore, for a "green" clustered cooperative system, we should target to improve UTs' EE-SE relationship by bringing the right balance among: 1) the cooperation effect; 2) the ICLI effect and; 3 ) the UTs power usage ${ }^{1}$. In the following, we analyse the feasible ICLI management techniques which aim to engage either separately or jointly these factors in an efficient way to improve the overall UTs' performance in the multi-cell system.

\section{INTER-CLUSTER INTERFERENCE MANAGEMENT}

In this section, we investigate efficient ways to suppress ICLI in the uplink of cooperative systems. We categorise ICLI management based on the way system resources are accessed among the clusters: a) Fully- or Partially-Orthogonal inter-cluster medium access through Spectrum Re-use; and b) Non-Orthogonal inter-cluster medium access through UT Power Control. We analyse both approaches to acquire EE-SE formulations; our main target is to identify under which conditions an ICLI management technique becomes the "greenest" solution, i.e. providing the highest improvement in overall end user devices' EE. We also show why partially-orthogonal intercluster medium-access techniques are suboptimal in terms of capacity compared to the fully-orthogonal one. Furthermore, we discuss shifting clustering as a potential solution to improve system fairness, in terms of per user performance.

\section{A. Spectrum Re-use}

1) Fully-Orthogonal access: First, we analyse the case where spectrum re-use achieves the total elimination of ICLI.

\footnotetext{
${ }^{1}$ Note that in (17), if the UT circuit power is much larger than the average transmit power, i.e. $Q K P_{C} \gg \sum_{q} \sum_{k} P_{q, k}$ (see also (14)), the total UTs power becomes independent of the transmit power vector, i.e. $\sum_{q} \sum_{k} P_{q, k}^{\mathrm{TOT}} \not \subset \mathbf{p}$. In that case, the EE is also maximised by $\mathbf{p}^{\star}$.
}

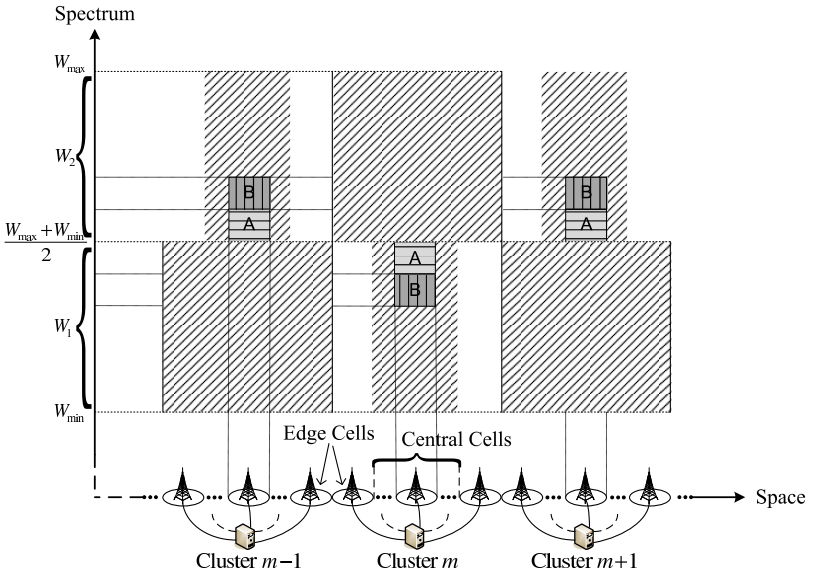

Fig. 2. Descriptive illustration of the two Spectrum Re-use techniques (example for $L=1$ ). With Spectral Isolation technique, central cells are allocated full system resources while adjacent edge cells orthogonally use bands $W_{1}$ and $W_{2}$ (in our case, $W_{1}=W_{2}=\frac{W_{\max }+W_{\min }}{2}$ ). By PartiallyOrthogonal Access, multiple spectral sub-bands, e.g. areas A and B, instead of the whole spectrum, can be allocated to cells (see also Appendix A).

We define this as the Spectral Isolation (SI) technique. Either frequency or time division approaches can be considered to isolate the clusters from each other. Without any loss of generality, we assume that a BS at any reference cell can "hear" users residing at a maximum of $L$ cells away from this reference cell, i.e. BSs can receive signals from UTs residing in their cell and in $L$ cells from each side. To efficiently take advantage of this maximum ICI span, we employ the orthogonal medium access only between neighbouring $L$ edge cells from each side of a cluster while letting all other central cells to use the full system resources. Therefore, BSs and UTs at the $2 L$ edge cells of each cluster are orthogonally using spectrum $W_{1}$ or $W_{2}$, with $W_{1}=W_{2}=\frac{W}{2}$, to respectively receive and transmit (see Fig. 2) and ICLI at the edges of each cluster is avoided.

The sum rate at any cluster, and therefore the corresponding UTs' EE, can be investigated separately for each spectrum sub-band. The average UT EE in that case will be given by:

$$
U_{\mathrm{SI}}=\frac{1}{W}\left(W_{1} U_{\mathrm{SI}}^{(1)}+W_{2} U_{\mathrm{SI}}^{(2)}\right),
$$

where $U_{\mathrm{SI}}^{(i)}$ is the average UT EE achieved at spectrum $W_{i}$. With no ICLI in the system, the maximum sum rate is achieved, as in the global cooperation case [25], when all UTs are always allowed to transmit with their maximum allowed power $P_{\max }$, i.e. $\mathbf{p}^{\star}=P_{\max } \cdot \mathbf{1}_{Q K}$. Based on (7) and (14), adopting the same approximation approach as for (10) and taking into consideration the cluster edge effects to create the $\mathbf{H H}^{\dagger}$ matrix we can find $U_{\mathrm{SI}}^{(i)}$ for any $Q \geq 2 L+1$ as:

$$
U_{\mathrm{SI}}^{(i)}=\frac{W \log _{2} \prod_{q \in \mathcal{Q}_{2}}\left[1+\frac{K P_{\max }}{\sigma^{2}} \sum_{\dot{q} \in \mathcal{Q}_{i}} \sum_{k=1}^{K}\left(\varsigma_{m, \dot{q}, k}^{m, q}\right)^{2}\right]}{Q K\left(P_{C}+P_{\max }\right)},
$$

where $\mathcal{Q}_{2}$ and $\mathcal{Q}_{1}$ denote the sets of all $Q$ cells and all $Q-2 L$ central cells of any cluster, respectively. Hence, the average UT EE will be given by replacing (19) in (18). 
2) Partially-Orthogonal Access: A question that legitimately arises is if the use of a partially-orthogonal mediumaccess spectrum re-use technique, i.e. splitting the spectrum band into more than two sub-bands and letting UTs and BSs of a cell to use a part of those resources, could improve the capacity of the system (see Fig. 2). The answer to this question is not trivial. By controlling the band of the spectrum allocated to the BS and UTs of each cell we not only decrease the undesired power received to each $\mathrm{BS}$ in the neighbouring clusters but also the total desired power received at BSs in the cluster of interest. Our target is to investigate what happens with the achievable sum rate given these two effects of simultaneous increase or decrease of desired and undesired received power. Since there is also not any Power-related gain induced in this case the same conclusion will apply for the UTs energy performance.

In Appendix $\mathrm{A}$ it is shown that the effect of allocating an extra sub-band to any cell $q$ of any cluster $m$ in the system will always be either positive or negative in terms of the achievable cluster sum rate. Therefore, the best strategy is to allocate either half (i.e. SI technique, to isolate clusters in case the negative effect of ICLI is dominant), or the whole (i.e. nonorthogonal access, to take advantage of the cooperation gain in case this effect is dominant) of the total available spectral resources to each cell.

\section{B. UT Power Control}

Unarguably, spectrum re-use techniques, although mitigating effectively ICLI, they are bandwidth wasting and essentially limit the achievable system SE. As an alternative solution, non-orthogonal multiple access techniques can be considered where UTs and BSs in all clusters are allowed to exploit the full spectrum available to the system and UT power control is employed to manage the ICLI. We define this as the Power Control (PC) approach. It should be also noted that in that case, where ICLI is present, allowing every UT to transmit with $P_{\max }$ does not necessarily maximise cluster sum rate anyway; in particular, recalling (10), it is obvious that sum rate is not a linear function of the transmit power profile. Therefore, an alternative $\mathbf{p}^{\star} \preceq P_{\max } \cdot \mathbf{1}_{Q K}$ may exist as a solution for the optimization problem stated in (16). Moreover, it becomes apparent that this solution, since it provides Power-related EE gain, will be "greener" regarding overall UTs' energy consumption. In the following we construct a mathematically tractable model for the overall UTs' transmit power profile which will help us to investigate efficient UT power control solutions that can improve the UTs' EE-SE relationship.

1) Piecewise Linear Combined Transmit Power Profile: Since we are interested on the ergodic performance of the system, we assume that a UT's location defines the average strength of its signal over a large enough time period. Therefore, UTs located close to the center of their respective a) cell (defined as cell location dependency) and b) cluster (defined as cluster location dependency), are able to contribute more on the cluster desired received power and at the same time cause less ICLI. In line with the observation above, two power vectors are introduced, i.e. $\mathbf{p}_{\text {cell }}$ and $\mathbf{p}_{\text {cluster, }}$ referring

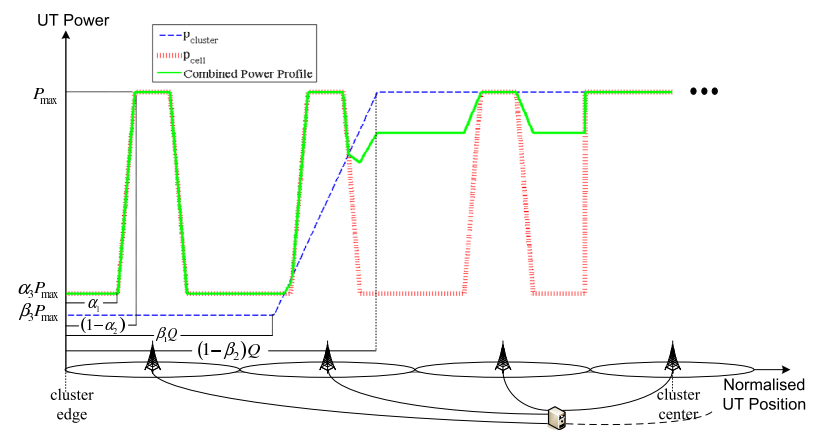

Fig. 3. Piecewise linear combined transmit power profile example; $Q=7$ cells forming a cooperation cluster with profile parameters: $\alpha_{1}=0.6, \alpha_{2}=$ $0.2, \alpha_{3}=0.1, \beta_{1}=\beta_{2}=0.4, \beta_{3}=0$ and $\nu=0.2$. X-axis is normalised over Inter-Site Distance.

respectively to the cell and cluster location dependency. Based also on insights from [33] that the cell location dependency has a dominant role, the following decision algorithm is introduced to model any heuristic transmit power profile $\mathbf{p}$ :

$$
\left\{\begin{array}{cl}
(\mathbf{p})_{j}=\left(\mathbf{p}_{\text {cell }}\right)_{j}, & \left(\mathbf{p}_{\text {cell }}\right)_{j} \geq\left(\mathbf{p}_{\text {cluster }}\right)_{j} \\
(\mathbf{p})_{j}=\nu\left(\mathbf{p}_{\text {cell }}\right)_{j}+\dot{\nu}\left(\mathbf{p}_{\text {cluster }}\right)_{j}, & \left(\mathbf{p}_{\text {cell }}\right)_{j}<\left(\mathbf{p}_{\text {cluster }}\right)_{j}
\end{array}\right\}
$$

where $(\cdot)_{j}$ refers to the $j^{\text {th }}$ element of the respective power vector, $\nu$ (with $0 \leq \nu \leq 1$ ) is a weighting parameter and $\nu=$ $1-\nu$. Furthermore, we introduce a mathematically tractable piecewise linear model with additional weighting parameters $\alpha_{1}, \alpha_{2}, \alpha_{3}$ for $\mathbf{p}_{\text {cell }}$ and $\beta_{1}, \beta_{2}, \beta_{3}$ for $\mathbf{p}_{\text {cluster }}$ to control the curve of each transmit power profile (see Fig. 3):

$$
\begin{aligned}
& 0 \leq \alpha_{1}, \beta_{1} \leq 1 \text { - edge-UTs with } P_{\min } \\
& 0 \leq \alpha_{2}, \beta_{2} \leq 1 \text { - center-UTs with } P_{\max } \\
& 0 \leq \alpha_{3}, \beta_{3} \leq 1 \text { - define } P_{\min }=\alpha_{3} P_{\max }, \beta_{3} P_{\max }
\end{aligned}
$$

with $\left(\alpha_{1}+\alpha_{2}\right),\left(\beta_{1}+\beta_{2}\right) \leq 1$. Terms edge- and center- refer to the respective location of the UT on either the cell or cluster.

We first focus on the power vector $\mathbf{p}_{\text {cell }}$. Since the power of a UT will be a function of distance $s$ from its respective BS, its elements should take values from the set:

$$
\left\{\begin{array}{cc}
P_{\min }=\alpha_{3} P_{\max }, & \frac{1}{2} \cdot \operatorname{ISD}\left(1-\alpha_{1}\right) \leq s \leq \frac{1}{2} \cdot \mathrm{ISD} \\
P(s), & \frac{1}{2} \alpha_{2} \cdot \operatorname{ISD} \leq s \leq \frac{1}{2} \cdot \operatorname{ISD}\left(1-\alpha_{1}\right) \\
P_{\max }, & 0 \leq s \leq \frac{1}{2} \alpha_{2} \cdot \operatorname{ISD}
\end{array}\right\}
$$

where power function $P(s)$ is defined as:

$$
P(s) \triangleq P_{\max }\left(\alpha_{3}+\left(1-\alpha_{3}\right) \frac{\left|s-\frac{1}{2} \operatorname{ISD}\left(1-\alpha_{1}\right)\right|}{\frac{1}{2} \operatorname{ISD}\left(1-\alpha_{1}-\alpha_{2}\right)}\right) .
$$

For the $\mathbf{p}_{\text {cluster }}$ power vector, the set of elements is obtained similarly as above with ISD and $\alpha$ parameters in (22) and (23) replaced by $Q \cdot$ ISD and $\beta$, respectively. It can be observed that the proposed transmit power profile requires the assignment of $P_{\max }$ to at least one UT in any cluster. This design decision originates from another important remark: "if a UT maximum power constraint exists, at least one element of the optimal transmit power profile $\mathbf{p}^{\star}$ must be equal to that constraint." The proof is provided in Appendix B. Furthermore, Fig. 
3 illustrates a descriptive example of the piecewise linear combined transmit power profile.

Therefore, based on (7) and (14), the average UT EE for the PC technique will be given by:

$$
U_{\mathrm{PC}}=\frac{W \log _{2} \prod_{q=1}^{Q}\left[1+\frac{\sum_{\dot{q}=1}^{Q} \sum_{k=1}^{K} P_{\dot{q}, k}(s)\left(\varsigma_{m, \dot{q}, k}^{m, q}\right)^{2}}{\sigma^{2}+\sum_{\dot{m}} \sum_{\dot{q}=1}^{Q} \sum_{k=1}^{K} P_{\dot{q}, k}(s)\left(\varsigma_{\dot{m}, \dot{q}, k}^{m, q}\right)^{2}}\right]}{Q K P_{C}+\sum_{q} \sum_{k} P_{q, k}(s)},
$$

where each UT power $P_{q, k}(s), \forall q, k$, is obtained from the piecewise linear combined transmit power profile according to the chosen weighting parameters.

\section{Shifting Clustering. A solution for Fairness}

In a "static" cellular system, where UTs rarely move to other cells, the UTs at edge cells from each cluster will "suffer" from the ICLI management techniques and they will be able to achieve less performance than the ones located at central cells. For a more balanced system with equally served UTs, a Shifting Clustering strategy could be considered for implementation where all the cells eventually acquire every possible position within the cluster for equal amount of time during a large period. This strategy would render possible for all UTs to achieve similar performance. Such shifting clustering could be achieved by either having the "active" backhaul links between the JPs and BSs change periodically with time or allowing JPs to connect with each other or to another processor so as clusters are virtually altered (i.e. the processors change the cells that are jointly processed) over time. In the linear system, at least $2 Q-1$ (instead of only $Q$ ) BSs need to be able to be connected (physically or virtually) to the same central processor in order for each cell to assume all possible positions within a cluster. Thus, on an overall system performance evaluation, one has to take into account the extra cost induced due to the increased backhauling needs. All in all, there will be a tradeoff between the extra induced cost and the advantage of achieving fairness in the sense of UTs performance. This study is of course out of the scope of this paper but a promising fairness solution is indicated here as an interesting direction for future research.

\section{Evaluation and Discussion}

This section aims to interpret the information theoretic results into a practical system scenario and evaluate UTs spectral and energy performance in the context of realworld cellular networks. To this end, an exemplar UMTSbased system model and propagation parameters suggested by 3GPP in [34] are chosen. Path loss coefficients are computed considering power loss $G_{0}$ at unit reference distance as: $\varsigma(d)=\sqrt{G_{0}}(1+d)^{-\eta / 2}$ while $G_{0}$ is fitted to the respective "Urban Macro - LOS" empirical scenario. Table I summarises the various system parameter values and ranges.

The approximation analytical results on UTs' EE-SE relationship (i.e. derived using (10)) have been validated through hybrid event-driven/Monte-Carlo simulations. Averaged $n u$ merical results (i.e. derived using (4)) were obtained from
TABLE I

SySTEM MODEl PARAMETERS

\begin{tabular}{|c|c|c|}
\hline Parameter & Symbol & Values \& Ranges \\
\hline Channel Bandwidth & $B$ & $5 \mathrm{MHz}$ \\
Thermal Noise Density at BS & $N_{0}$ & $-169 \mathrm{dBm} / \mathrm{Hz}$ \\
UTs per Cell & $K$ & $\{20,100\}$ \\
Inter Site Distance & ISD & $100 \mathrm{~m}$ to $5 \mathrm{Km}$ \\
Reference Distance & $d_{0}$ & $1 \mathrm{~m}$ \\
Power Loss at Reference Distance & $G_{0}$ & $-34.5 \mathrm{~dB}$ \\
Path Loss Exponent & $\eta$ & $\{2,3,3.5\}$ \\
UT Max Transmit Power & $P_{\max }$ & $23 \mathrm{dBm}$ \\
Average UT Circuit Power & $P_{\mathrm{C}}$ & $20 \mathrm{dBm}$ \\
Cooperation Cluster Size & $Q$ & 1 to 7 \\
\hline
\end{tabular}

generating 100 random system instances to construct the system channel matrices at each instance. More specifically, at each system instance, the BSs-UTs distances were calculated by uniformly placing the UTs across the system grid. At the same instance, Rayleigh fading coefficients were generated for all UT-BS links in the network.

\section{A. Simulation Setup and Initial Observations}

Here, we aim to define the scenarios of interest and narrow down the focus of our research on the most beneficial cases regarding system performance. The first inquisition appertains the system topology and the propagation environment. The urban macro system is a multi slope environment which means that the path loss exponent highly depends on the UT-BS distances [14], [35]. Therefore, since the ISD relates directly with the average UT-BS distances, it is safe to define the system density according to the combination of ISD and $\eta$ system parameters. Three representative system density scenarios are defined and examined in the following: 1) "Dense" (ISD $=100 \mathrm{~m}, \eta=2)$; "Medium" (ISD=600m, $\eta=3)$; and 3) "Sparse" $(\mathrm{ISD}=2 \mathrm{Km}, \eta=3.5)$. Moreover, low values of $L$ (maximum ICI span) are considered (i.e. $L=1$ or $L=2$ ) since few strong interferers are expected in the examined urban macro system. In fact, this expectation was verified through the simulations where it was observed that, for all examined density scenarios, considering values of $L>2$ in both SI and $\mathrm{PC}$ techniques overestimates the amount of strong interferers and leads to suboptimal system performance.

Furthermore, an exhaustive search was performed to narrow down the best performing transmit power profiles for the PC technique. It was recognised that it is always more significant for the UTs to manage their power according to their cell location dependency. It was also observed that the most efficient strategy is to allow few "best" channel UTs in each cell to transmit at $P_{\max }$, while advising most of the rest to use $P_{\min }$ during that communication slot. This observation comes in agreement with the mathematical proof in Appendix B, suggesting that at least one element of the sum rate maximising vector $\mathbf{p}^{\star}$ must be equal to $P_{\max }$. Thus, in the following we mainly concentrate on this advantageous cell-based opportunistic transmission strategy with weighting parameters $v=1, \alpha_{1}=0.45$ and $\alpha_{2}=0.05$. Note that this transmission strategy is more applicable to an elastic traffic case, for delay tolerant applications, e.g. data transfer through internet access. 


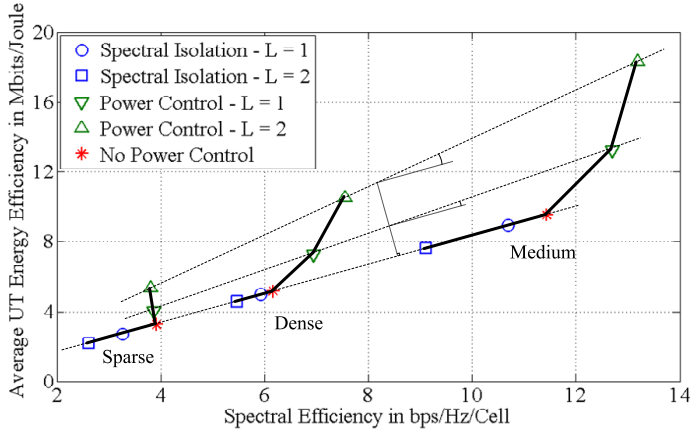

Fig. 4. Effect of inter-cluster interference management. UTs' EE vs. system SE for various ICLI management techniques and system density scenarios. $Q=6, K=20, P_{\max }=200 \mathrm{~mW}, \alpha_{3}=0$.

\section{B. Results and Evaluation}

In light of the above insights, we begin the evaluation of the overall UTs' EE-SE performance for the different ICLI management techniques while investigating the pragmatic effect of the various system parameters.

1) General Comparison of ICLI Management Techniques: Firstly, in Fig. 4 we compare the performance achieved by the SI and the PC techniques for different system density scenarios. In addition, we examine the benchmark case of $N o$ Power Control (NPC) where UTs and BSs in all clusters use the full spectrum available to the system and all UTs transmit with $P_{\max }$ causing the maximum possible ICLI. We consider cooperation clusters of size $Q=6$ and for fair comparisons we assume that power control in the PC technique is performed only for UTs in the $2 L$ edge cells of each cluster, while letting all other UTs in central cells transmit with $P_{\max }$. To this end, Fig. 4 depicts that SI technique leads to a suboptimal overall performance when compared to NPC technique for every examined system density scenario. This behaviour shows that, for these scenarios, the combined positive effect from ICLI management and negative effect from less bandwidth usage in SI technique leads to a performance loss which is lower than the one perceived from no ICLI management in NPC technique. In fact, it was observed during the simulations that SI could provide SE gain only in extremely dense deployments (e.g. for $\eta=2$, ISD $<100 \mathrm{~m}, K>100$ ) which however do not map to realistic macrocell scenarios. Moreover, it is observed that lower maximum ICI span (i.e. $L=1$ instead of $L=2$ ) is preferable when the SI technique is adopted. This implies that cooperation effect is stronger than ICLI effect in that case.

On the other hand, we observe that PC significantly outperforms the other techniques in dense and medium density systems. At the same time, for higher maximum ICI span (i.e. $L=2$ instead of $L=1$ ) overall UTs' performance is further improved. This is due to the fact the efficient UTs' power control keeps the cooperation effect vigorous while managing ICLI effectively. Specifically for dense systems, however, ICLI becomes considerably large, demeaning the impact of the cooperation effect. Therefore, medium density system seems to be the most viable scenario in general for implementation of clustered BS cooperation. In sparse scenarios, both cooperation and ICLI effects become rather insignificant and

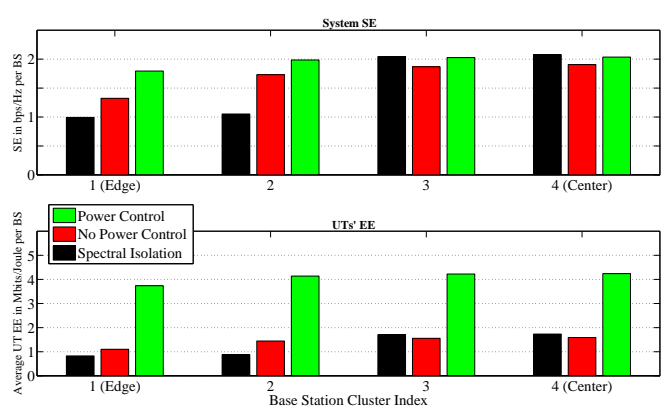

Fig. 5. Average BS contribution on system SE and UTs' EE for the various ICLI management techniques. $Q=7$, ISD $=1 \mathrm{Km}, \eta=3, K=20$. For SI: $L_{\max }=2$. For PC: $P_{\max }=200 \mathrm{~mW}, \alpha_{3}=0$ for all cells.

thus, any attempt to manage ICLI will generally decrease the $\mathrm{SE}$ of the system. For that reason it is depicted in that case that PC technique, although still provides a slightly better EE performance, it cannot achieve the SE of the NPC technique. Of course, it should be mentioned here that an operator should also consider the cost of employing cooperation and/or ICLI management along with the expected performance gain of each technique in order to reach to the most profitable solution.

2) Individual BS Performance Contribution: A more complete view on the UTs' EE-SE performance under different ICLI management techniques is attained in Fig. 5. Focusing on the advantageous medium density scenario, we examine the average per cell SE and UT EE contributed by each cooperating BS for the case of $Q=7$. Regarding the "greenness" of each technique, it is evident here again that $\mathrm{PC}$ is by far the highest performing one due to its implicit energy-saving nature. However, an additional important insight is perceived by this figure which can prove particularly useful in case operators are required to optimize performance via BS deployment. Under SI technique, BSs in central cells contribute more on overall system performance than BSs in edge cells; on the other hand, a more "fair" performance contribution from each BS in the cluster is attained under $\mathrm{PC}$ technique due to the fact that ICLI management through this technique leads to similar average desired and undesired received power to all BSs in the cluster (see (11)). Thus, while no particular care on deployment is needed when PC technique is adopted, in cases where SI technique can be optimal (e.g. dense small cell networks), careful selection or even re-deployment of BSs to form clusters may provide further boost on overall performance.

3) Effect of UT power control: Having established the superiority of the PC technique on the system scenarios of interest, we attempt to obtain a closer look on this specific ICLI management technique. Fig. 6 illustrates the UTs' EESE relationship for various transmit power profiles following the cell-based opportunistic transmission strategy. Results for the three representative system density scenarios and for various cluster sizes (i.e. $Q=1$ to 6 ) are obtained and also compared with the NPC technique. We should note that the case of $Q=1$ corresponds to the conventional case of $\mathrm{No}$ Cooperation among cells where similar UT power control based techniques are used for managing ICI. This is hereafter 


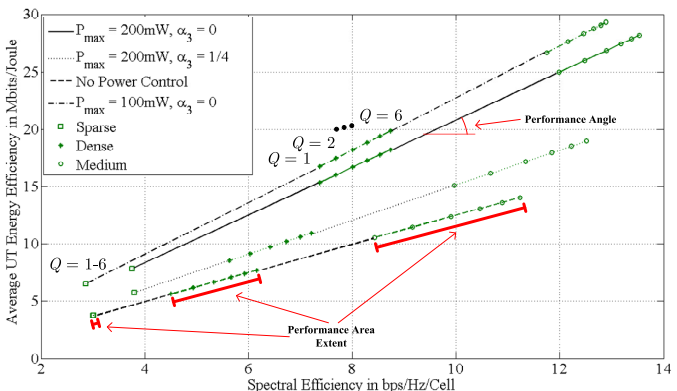

Fig. 6. Effect of ICLI management via UT power control on UTs' EE-SE relationship for various density systems and cluster sizes $(K=20)$. Different transmit power profiles alter the Performance Angle (i.e. Power-related gain) and the Performance Area Extend (i.e. SE-related gain due to cooperation) of the linear relationship.

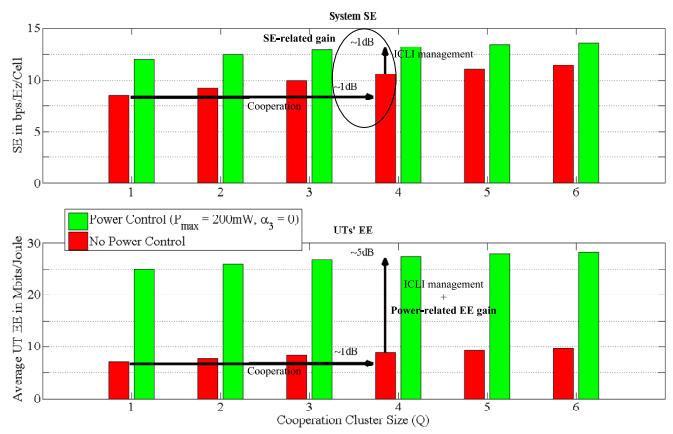

Fig. 7. SE-related vs. Power-related gain under PC ICLI management technique. Effect of cooperation and UT power control on System SE and UTs' EE gains. $K=20$, ISD $=1 \mathrm{Km}, \eta=3$.

defined as the NC-PC technique.

To this end, in Fig. 6, it is observed that when UTs follow a cell-based transmission strategy, their EE-SE relationship for any $Q$ remains linear, as expected considering (14). Following a different transmit power profile changes: 1) the Power-related gain and thus, alters the Performance Angle of the linear EESE relationship (i.e. higher angle for lower overall UTs' power consumption and vice versa) as well as; 2) the SE-related gain due to cooperation defined as Performance Area Extent in that case. Note also that, without considering the channel estimation overhead, more cooperation (in the sense of larger cluster sizes) leads to higher overall performance. In particular, it is observed that larger $Q$ provides higher SE-related EE gain due to cooperation (i.e. higher performance area extent) in the medium density scenario while there is not any actual gain in the sparse scenario. Finally, it is noted that for systems with relaxed SE requirements, higher Power-related EE gains may be achieved when the best channel UTs transmit with less than the actual maximum available power, while the rest UTs remain "silent" during the specific transmission slot. For example, in the same figure, it is depicted that with $P_{\max }=100 \mathrm{~mW}(<200 \mathrm{~mW})$ and $P_{\min }=0$, high UTs' EE can be achieved with minor system SE reduction, while the choice of $P_{\min }=50 \mathrm{~mW}$ significantly degrades the system overall performance.

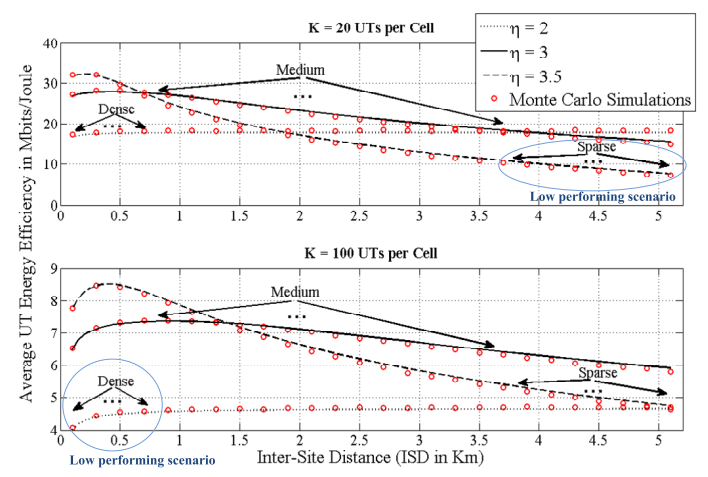

Fig. 8. Effect of System density and UT density on UTs' EE. $Q=3$, $P_{\max }=200 \mathrm{~mW}, \alpha_{3}=0$. Arrows fuzzily denote the system density areas of practical interest.

4) Cooperation versus ICLI Management Gain: In order to quantify and compare the performance gains due to cooperation and UT power control in the PC technique, we plot system SE and average UT EE versus various cluster sizes $(Q=1$ to 6$)$ for the medium density scenario in Fig. 7. While the improvement in SE is quite decent and equally originates from both cooperation and ICLI management, we attain a considerable EE gain due to the efficient UTs' power control. A numerical example is given when comparing the performance achieved under NC-PC and NPC techniques to the one of PC technique with cooperating clusters of $Q=4$ cells. In that case, it is depicted that system SE perceives $1 \mathrm{~dB}$ gain due to cooperation plus $1 \mathrm{~dB}$ gain due ICLI management through power control. On the other hand, UTs' EE perceives the same gain from cooperation (i.e. SE-related EE gain) but in addition, there is a much more significant gain of $5 \mathrm{~dB}$ introduced from UTs' power control (i.e. Power-related EE gain). This highlights the significance of the opportunistic transmission strategy on the improvement of users' overall performance in a clustered cooperative system suffering from the negative effect of ICLI.

5) Effect of System and UT density: Finally, we aim to shed more light on the effect of system density (combined ISD and $\eta$ effect) and UT density on the UTs' EE when PC technique is employed. To this end, the "Dense", "Medium" and "Sparse" system density scenario are redefined to include areas of practical interest (i.e. a viable combination of path loss exponents with ISD ranges) and are fuzzily denoted in Fig. 8. It becomes apparent, more clearly now, that medium density systems are beneficial for increasing UTs' EE through clustered BS cooperation. Furthermore, regarding UT density, by comparing upper (lower UT density) and bottom (higher UT density) graphs in Fig. 8 we observe that lower/higher number of UTs per cell renders denser/sparser systems more viable for implementation of clustered BS cooperation and vice versa. This phenomenon takes place in the examined macrocell scenario because higher/lower UT density amplifies more/less the ICLI effect than the cooperation effect. It is also noted that Fig. 8 reveals the close match between analytical and numerical results verifying the validity of the approximated EE-SE formulations arising from theoretical analysis. 
In general, the above observations give in combination a very important indication to network engineers; cooperation schemes should be implemented with care according to the expected system deployment. In real network with variable system and UT density over space and time, adaptive cooperation mechanisms could make sure that appropriate cooperation clusters and ICLI management techniques are jointly chosen so as to always lie in the desired EE-SE performance area.

\section{CONCLUSION}

In this work, we have investigated inter-cluster interference management techniques to improve user EE-SE relationship in uplink of clustered cooperative cellular systems. Our investigations focused on the potential Bit-per-Joule gains for all users while at the same time the highest possible gains on ergodic system sum rate are obtained. For this, we have tried to find which technique, and under what circumstances, gives a good balance between the harmful effect due to ICLI and the beneficial effect originating from joint signal processing at cooperating BSs.

Through the analysis, two main types of performance gain were acknowledged, namely the SE-related gain due to the efficient blend of cooperation and ICLI management and the Power-related gain due to efficient power usage at users end. Furthermore, two main feasible and effective ICLI management techniques were identified: 1) the SI technique, based on orthogonal resource splitting among clusters to eliminate ICLI; and 2) the PC technique, based on an efficient cell-based opportunistic transmission power control strategy at UTs, where only few UTs every time take advantage of their strong channel conditions with their serving BS, to optimize the Power-related gain while mitigating ICLI. These techniques were investigated in detail and compared with each other as well as with the conventional case of no cooperation and the scenario of clustered cooperation with total interference allowance. In addition, various key design parameters that affect users' performance have been identified and their effect is examined in detail. Specifically, these parameters are the inter-site distance; path loss exponent; number of served UTs per cell; UTs transmit powers; and cooperation cluster size.

Based on the investigations it was established that the PC technique can significantly improve the energy and spectral efficiency of the users for dense to medium density system deployments. In fact, the medium density system, which is the typical region of operation for macrocell networks, proves to provide the best SE-related gain under clustered BS cooperation while even more significant EE gains at user terminals are achieved through overall users power control. At the same time, lower/higher number of UTs per cell can render denser/sparser deployments more viable for implementation of clustered BS cooperation and vice versa. In addition, it was noted that when SE requirements are relaxed, higher Powerrelated gain may be achieved if, during a specific transmission slot, the best channel UTs transmit with lower than the maximum available power while the rest UTs remain silent. On the other hand, the SI technique can only provide SErelated gains in very dense systems and thus, could be deemed effective for pico- or femto- cellular deployments. However, higher cost and more complex operations may be needed for achieving ubiquitous performance across such clustered systems; in fact, through the introduction of the BS performance contribution metric, it was observed that adaptive selection of BSs to form highly isolated clusters may provide further boost on overall performance in such scenarios. In very sparse systems, where the effects of cooperation and ICLI become subtle, any attempt for interference management to improve EE-SE relationship becomes rather irrelevant and thus, low complexity non-cooperative schemes should be preferred.

We finally note that in this paper the EE gains are from the UTs point of view. The important aspect of energy consumption at the UT side attributes to both overall energy consumption of the network as well as the battery longevity of the user devices and it is a significant system design target. The proposed ICLI management techniques could be used in a complementary way with other popular techniques for energy savings at user devices such as load balancing and computational offloading. In order to obtain a view on overall systems energy efficiency, we can additionally consider the extra backhaul and signal processing energy needs due to higher cooperation size. This is an interesting direction for future research.

\section{APPENDIX A}

\section{SUB-OPTIMALITY OF PARTIALLY-ORTHOGONAL MEDIUM-ACCESS SPECTRUM RE-USE}

In this appendix we show that for a linear clustered system using spectral isolation for ICLI management, there is only need of a total of two sub-bands to be allocated to the BS and UTs of each cell.

Consider a system with $Q$ cells per cluster, total available spectrum band $W$ and an even number of total $2 X$ (with $X>1$ ) equal spectrum sub-bands (see Fig. 2). In general, the cluster ergodic sum rate will be the sum of achievable rates $R_{m}^{(x)}$ at each spectrum sub-band $W_{x}=\frac{W}{2 X}$ :

$$
R_{m}=\frac{1}{W} \sum_{x} W_{x} R_{m}^{(x)}, \forall x \in\{1 \ldots 2 X\} .
$$

Allocating half of the spectrum to each cluster, the achievable sum rate of a cluster $m$ will be

$$
R_{m}=\frac{1}{W} \sum_{x=1}^{X} W_{x} R_{m}^{(x)}=\frac{1}{W} \sum_{x=X+1}^{2 X} W_{x} R_{m}^{(x)},
$$

since the BSs of cluster $m$ do not operate in bands $W_{i}, \forall i>$ $X$. Let us now allocate band $W_{X+1}$ to cell $q$ of cluster $m$. Due to the symmetrical nature of the system, in neighbouring clusters $m-1$ and $m+1$, the respective cells $q$ will be allocated with the extra band $W_{X}$. The new band allocation is illustrated in Fig. 2 (shaded areas A). The $m$-th cluster sum rate in that case will be given by:

$$
\dot{R}_{m}=\frac{1}{W}\left(\sum_{x=1}^{X-1} W_{x} R_{m}^{(x)}+W_{X} \dot{R}_{m}^{(X)}+W_{X+1} \dot{R}_{m}^{(X+1)}\right)_{(27)}
$$


The allocation of the extra sub-band $X+1$ at cell $q$ of cluster $m$ brought an additional term in (27), i.e. $\frac{W_{X+1}}{W} \dot{R}_{m}^{(X+1)}$, which is due to the increase on the desired received power in the cluster. At the same time though, the allocation of sub-band $W_{X}$ in cells $q$ of clusters $m-1$ and $m+1$ results into intercluster interference (or undesired received power ) for the BS in cell $q$ of cluster $m$ which already operates at that sub-band leading to a decrease on achievable rate at sub-band $W_{X}$, i.e. $\dot{R}_{m}^{(X)}<R_{m}^{(X)}$.

We define the rate differences:

$$
\begin{aligned}
& \delta \dot{R}_{\text {undesired }} \triangleq R_{m}^{(X)}-\dot{R}_{m}^{(X)}>0 \\
& \delta \dot{R}_{\text {desired }} \triangleq \dot{R}_{m}^{(X+1)}>0 \\
\text { and } & \delta \dot{R}_{m} \triangleq \delta \dot{R}_{\text {desired }}-\delta \dot{R}_{\text {undesired }}
\end{aligned}
$$

with $\delta \dot{R}_{m}$ essentially reflecting the difference between $R_{m}$ and $\dot{R}_{m}$. Apparently, if the allocation of the extra sub-band has an increasing effect on the achievable sum rate of cluster $m, \delta \dot{R}_{m}$ will result into a positive value, and vice versa.

Now, compared to the previous case where sub-band $W_{X+1}$ (and $W_{X}$, respectively) was allocated to cell $q$ of cluster $m$ (clusters $m-1$ and $m+1$, respectively), let us allocate subband $W_{X+2}$ (and $W_{X-1}$, respectively) to the same cell $q$ of cluster $m$ (clusters $m-1$ and $m+1$, respectively). This additional band allocation is illustrated by the shaded areas $\mathrm{B}$ in Fig. 2. The cluster $m$ sum rate becomes:

$$
\begin{array}{r}
\ddot{R}_{m}=\frac{1}{W}\left(\sum_{x=1}^{X-2} W_{x} R_{m}^{(x)}+W_{X} \ddot{R}_{m}^{(X-1)}+W_{X} \dot{R}_{m}^{(X)}\right. \\
\left.+W_{X+1} \dot{R}_{m}^{(X+1)}+W_{X+2} \ddot{R}_{m}^{(X+2)}\right),
\end{array}
$$

where, similarly as before, the extra term $\frac{W_{X+2}}{W} \ddot{R}^{(X+2)}$ comes due to the increased desired power in cluster while $\ddot{R}^{(X-1)}$ is related to the arising inter-cluster interference in subband $W_{X-1}$. A very important observation is that, in our symmetrical system, the extra allocation of $W_{X+2}$ sub-band in cell $q$ of cluster $m$, the additional desired and undesired power (and hence, the sum rate) in cluster $m$ will be the same as in the previous case (when $W_{X+1}$ was allocated), i.e.

$$
\ddot{R}^{(X+2)}=\dot{R}_{m}^{(X+1)}
$$

and

$$
R^{(X-1)}-\ddot{R}^{(X-1)}=R_{m}^{(X)}-\dot{R}_{m}^{(X)}
$$

leading to:

$$
\begin{aligned}
& \delta \ddot{R}_{m} \triangleq \ddot{R}_{m}^{(X+2)}-\left(R_{m}^{(X-1)}-\ddot{R}_{m}^{(X-1)}\right) \\
&=\dot{R}_{m}^{(X+1)}-\left(R_{m}^{(X)}-\dot{R}_{m}^{(X)}\right)=\delta \dot{R}_{m} .
\end{aligned}
$$

where $\delta \ddot{R}$ essentially reflects the difference between $\dot{R}_{m}$ and $\ddot{R}_{m}$. Hence, the effect of allocating an extra sub-band to any cell $q$ of any cluster $m$ in the system will always be either positive or negative in terms of the achievable cluster sum rate. That means that the allocation of more sub-bands to cells' BSs and UTs can only either increase or decrease the achievable cluster sum rate. Since there is also not any Power-related gain induced in this isolation case (i.e. UTs transmit with $P_{\max }$ for achieving maximum sum rate) the same conclusion applies for the energy performance of the system.

\section{APPENDIX B}

\section{ON THE OPTIMAL UT POWER PROFILE}

We extend a technique presented in [36] to narrow down the possible solutions of (16). Given a real factor $\varepsilon>1$ and an overall UTs' transmit power profile $\mathbf{p}$, we have from (12) that

$$
\begin{aligned}
& R_{m, q}(\varepsilon \mathbf{p}) \triangleq R_{m, q}\left(\varepsilon P_{1,1}, \ldots, \varepsilon P_{Q, K}\right) \\
& =W \log _{2}\left(1+\frac{\sum_{\dot{q}=1}^{Q} \sum_{k=1}^{K} P_{\dot{q}, k}\left(\varsigma_{m, \dot{q}, k}^{m, q}\right)^{2}}{\frac{\sigma^{2}}{\varepsilon}+\sum_{\dot{m}} \sum_{\dot{q}=1}^{Q} \sum_{k=1}^{K} P_{\dot{q}, k}\left(\varsigma_{\dot{m}, \dot{q}, k}^{m, q}\right)^{2}}\right) \\
& >R_{m, q}(\mathbf{p})
\end{aligned}
$$

for all BSs $q$ and any cluster size $Q$. Since the total cluster sum rate is $R_{m}(\varepsilon \mathbf{p})=\sum_{q=1}^{Q} R_{m, q}(\varepsilon \mathbf{p})$, we have also that

$$
R_{m}(\varepsilon \mathbf{p})>R_{m}(\mathbf{p}) \text {. }
$$

\section{REFERENCES}

[1] G. P. Fettweis and E. Zimmermann, "ICT Energy Consumption - Trends and Challenges," in 11th International Symposium on Wireless Personal Multimedia Communications (WPMC'08), Lapland, Finland, Sep. 2008.

[2] G. Auer, I. Godor, L. Hevizi, M. A. Imran, J. Malmodin, P. Fazekas, G. Boczok, D. Zeller, O. Blume, and R. Tafazolli, "The EARTH Project: Towards Energy Efficient Wireless Networks," ICT Future Network and Mobile Summit, June 2010.

[3] Z. Hasan, H. Boostanimehr, and V. Bhargava, "Green cellular networks: A survey, some research issues and challenges," Communications Surveys Tutorials, IEEE, vol. 13, no. 4, pp. 524-540, 2011.

[4] T. Chen, Y. Yang, H. Zhang, H. Kim, and K. Horneman, "Network energy saving technologies for green wireless access networks," Wireless Communications, IEEE, vol. 18, no. 5, pp. 30-38, 2011.

[5] T. Han and N. Ansari, "On greening cellular networks via multicell cooperation," Wireless Communications, IEEE, vol. 20, no. 1, pp. 8289, 2013.

[6] N. Balasubramanian, A. Balasubramanian, and A. Venkataramani, "Energy Consumption in Mobile Phones - A Measurement Study and Implications for Network Applications," in Internet Measurement Conference, Nov. 2009.

[7] P. Somavat, S. Jadhav, and V. Namboodiri, "Accounting for the Energy Consumption of Personal Computing Including Portable Devices," in e-Energy '10, Passau, Germany, 2010.

[8] Cisco, "Cisco Visual Networking Index: Global Mobile Data Traffic Forecast Update, 2012-2017," Tech. Rep., February 2013.

[9] A. Lozano, J. Heath, R., and J. Andrews, "Fundamental limits of cooperation," Early Access Article in IEEE Transactions on Information Theory, 2013.

[10] M. Karakayali, G. Foschini, and R. Valenzuela, "Network coordination for spectrally efficient communications in cellular systems," Wireless Communications, IEEE, vol. 13, no. 4, pp. 56-61, Aug. 2006.

[11] O. Somekh, O. Simeone, Y. Bar-Ness, A. Haimovich, U. Spagnolini, and S. Shamai, "An information theoretic view of distributed antenna processing in cellular systems," in ch.2, pp.31-64, Distributed Antenna Systems: Open Architecture for Future Wireless Communications, H. Hu, Y. Zhang, and J. Luo, Eds. Auerbach Publications, CRC Press, 2007.

[12] H. Dai and H. Poor, "Asymptotic spectral efficiency of multicell MIMO systems with frequency-flat fading," IEEE Trans. Signal Processing, vol. 51, no. 11, pp. 2976-2988, Nov. 2003.

[13] M. Karakayali, G. Foschini, and R. Valenzuela, "Network coordination for spectrally efficient communications in cellular systems," Wireless Communications, IEEE [see also IEEE Personal Communications], vol. 13, no. 4, pp. 56-61, Aug. 2006.

[14] 3GPP - STG RAN, E-UTRA, "Further Advancements for E-UTRA, Physical Layer Aspects (Release 9)," 3GPP TR 36.814 v1.5.1 (200912), Tech. Rep., 2009. 
[15] A. Papadogiannis, D. Gesbert, and E. Hardouin, "A Dynamic Clustering Approach in Wireless Networks with Multi-Cell Cooperative Processing," in IEEE International Conference on Communications, ICC '08, May 2008.

[16] S. Venkatesan, "Coordinating Base Stations for Greater Uplink Spectral Efficiency in a Cellular Network," in IEEE 18th International Symposium on Personal, Indoor and Mobile Radio Communications, PIMRC '07, Sept. 2007.

[17] M. Sawahashi, Y. Kishiyama, A. Morimoto, D. Nishikawa, and M. Tanno, "Coordinated multipoint transmission/reception techniques for LTE-advanced [Coordinated and Distributed MIMO]," Wireless Communications, IEEE, vol. 17, no. 3, pp. 26-34, June 2010.

[18] R. Irmer, H. Droste, P. Marsch, M. Grieger, G. Fettweis, S. Brueck, H.-P. Mayer, L. Thiele, and V. Jungnickel, "Coordinated multipoint: Concepts, performance, and field trial results," Communications Magazine, IEEE, vol. 49, no. 2, pp. 102-111, February 2011.

[19] A. Fehske, P. Marsch, and G. Fettweis, "Bit per Joule efficiency of cooperating base stations in cellular networks," in IEEE GLOBECOM Workshops, Dec. 2010.

[20] 3GPP - UMTS, LTE, "Feasibility study for evolved Universal Terrestrial Radio Access (UTRA) and Universal Terrestrial Radio Access Network (UTRAN)," 3GPP TR 25.912 v8.0.0 (2009-01), Tech. Rep., 2009.

[21] Y. Chen, S. Zhang, S. Xu, and G. Li, "Fundamental Trade-offs on Green Wireless Networks," IEEE Communications Magazine, vol. 49, no. 6, pp. 30-37, June 2011

[22] G. Miao, N. Himayat, and G. Li, "Energy-efficient link adaptation in frequency-selective channels," IEEE Transactions on Communications, vol. 58 , no. 2, pp. 545-554, February 2010.

[23] M. Bacha, J. Evans, and S. Hanly, "On the Capacity of MIMO Cellular Networks with Macrodiversity," in 7th Australian Communications Theory Workshop, Feb. 2006.

[24] A. D. Wyner, "Shannon-Theoretic Approach to a Gaussian Cellular Multiple-Access Channel," IEEE Transactions on Information Theory, vol. 40, no. 6, pp. 1713-1727, Nov. 1994.

[25] O. Somekh and S. Shamai, "Shannon-Theoretic Approach to a Gaussian Cellular Multiple-Access Channel with Fading," IEEE Transactions on Information Theory, vol. 46, no. 4, pp. 1401-1425, 2000.

[26] S. Chatzinotas, M. Imran, and C. Tzaras, "The effect of user distribution on a linear Cellular Multiple-Access Channel," in Third International Conference on Communications and Networking in China. ChinaCom 2008, Aug. 2008.

[27] P. Marsch and G. P. Fettweis, Eds., Coordinated Multi-Point in Mobile Communications - From Theory to Practice. Cambridge University Press, 2011.

[28] N. A. Letzepis, "Gaussian cellular multiple access channels," Ph.D. dissertation, Institute for Telecommunications Research, University of South Australia, Dec. 2005.

[29] A. Motahari and A. Khandani, "Capacity Bounds for the Gaussian Interference Channel," IEEE Transactions on Information Theory, vol. 55, no. 2, pp. 620-643, Feb. 2009.

[30] S. Hanly and P. Whiting, "Information-theoretic capacity of multireceiver networks," Telecommunication Systems, vol. 1, pp. 1-42, 1993, 10.1007/BF02136153.

[31] T. M. Cover and J. A. Thomas, Elements of Information Theory 2nd Edition, 2nd ed. Wiley-Interscience, July 2006.

[32] S. Boyd and L. Vandenberghe, Convex Optimization. Cambridge University Press, 2004.

[33] E. Katranaras, D. Kaltakis, M. Imran, and R. Hoshyar, "Interference Allowance in Clustered Joint Processing and Power Allocation," 6th International Wireless Communications \& Mobile Computing Conference, (IWCMC'10), 2010.

[34] ETSI TR 125996 V7.0.0, "Universal Mobile Telecommunications System (UMTS); Spatial Channel Model for Multiple Input Multiple Output (MIMO) Simulations (3GPP TR 25.996 version 7.0.0 Release 7) ," June 2007

[35] E. Katranaras, M. Imran, and C. Tzaras, "Uplink Capacity of a Variable Density Cellular System with Multicell Processing," IEEE Transactions on Communications, vol. 57, no. 7, pp. 2098-2108, July 2009.

[36] C. S. Chen and G. Oien, "Optimal power allocation for two-cell sum rate maximization under minimum rate constraints," in IEEE International Symposium on Wireless Communication Systems, ISWCS '08, Oct 2008.

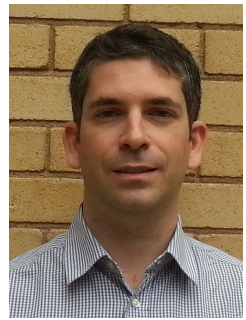

Efstathios Katranaras (M'06) received his B.Sc. degree in electrical and computer engineering from National Technical University of Athens, Greece, in 2005 and his M.Sc. (Distinction) and Ph.D. degrees in mobile communications from University of Surrey, UK, in 2006 and 2009, respectively. Dr. Katranaras is currently a Research Fellow in the Institute for Communication Systems (ICS) at the University of Surrey and an active research member for the 5G innovation centre (5GIC) at Surrey. His main research interests include the evaluation of fundamental capacity limits, energy efficiency in cellular systems and advanced multi-cell cooperation techniques for future dense small cell networks.

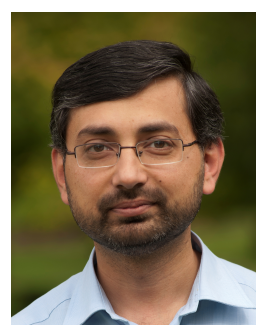

Muhammad Ali Imran (M'03, SM'12) received his M.Sc. (Distinction) and Ph.D. degrees from Imperial College London, UK, in 2002 and 2007, respectively. He is currently a Reader in the Institute for Communication Systems (ICS) at the University of Surrey, UK. In this role, he is leading a number of multimillion international research projects encompassing the areas of energy efficient design of cellular system, information theoretic performance limits, sensor networks and learning/self-organising techniques for optimisation of cellular system operation. He is also leading the new physical layer work area for $5 \mathrm{G}$ innovation centre at Surrey. He has a global collaborative research network spanning both academia and key industrial players in the field of wireless communications. He has supervised 17 successful $\mathrm{PhD}$ graduates and published over 150 peerreviewed research papers including more than 20 IEEE Journals. Dr. Imran is a senior member of IEEE and a Fellow of Higher Education Academy (FHEA), UK. He received Faculty Learning and Teaching Award 2014 and IEEE Communications Society's Fred W. Ellersick Prize.

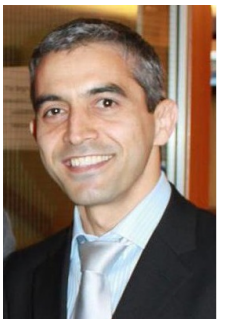

Mehrdad Dianati received his BSc. and MSc. both in Electrical Engineering from Sharif and K.N.T University of Technology, Iran. He completed his $\mathrm{PhD}$ in Electrical and Computer Engineering at the University of Waterloo in Canada. He is currently a Reader in Communications and Networking Systems. His research area includes: 1) analysis and design of advanced cross layer optimised PHY/MAC layer techniques for $5 \mathrm{G}$ wireless access networks, and 2) communication network design for Intelligent Transport Systems. He has been involved in a number of international projects in the area of communications and networking as technical coordinator and work-package leader. In addition to his research experience, Mehrdad has 9 years of industrial as software/hardware developer and technical leader. Dr. Dianati is an associate editor for IEEE Transactions on Vehicular Technology, IET Communications and Wiley's Journal of Wireless Communications and Mobile. He has also been actively involved in organizing committees of numerous international conferences in recent years.

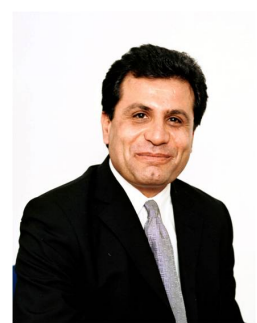

Rahim Tafazolli is the Director of the Institute for Communication Systems (ICS) and the 5G Innovation Center (5GIC) at the University of Surrey in the UK. He has published more than 500 research papers in refereed journals, international conferences and as invited speaker. He is the editor of two books on Technologies for Wireless Future published by Wileys Vol.1 in 2004 and Vol.2 2006. He is currently chairman of EU Net!Works Technology Platform Expert Group, board member of the UK Future Internet Strategy Group (UK-FISG). Prof. Tafazolli was appointed as Fellow of WWRF (Wireless World Research Forum) in April 2011, in recognition of his personal contribution to the wireless world. As well as heading one of Europe's leading research groups. 